\title{
Neuroendocrine carcinoma of the ureter: A case report and literature review
}

\author{
WEI WANG ${ }^{1}$, GUANGMING LIU $^{1}$, YANMIN LI $^{2}$, UDESHIKA SIRIWARDANE $^{3}$ and HONGSHUN MA ${ }^{1}$ \\ Departments of ${ }^{1}$ Urology and ${ }^{2}$ Pathology, Tianjin First Central Hospital, Tianjin 300192; \\ ${ }^{3}$ International Medical School, Tianjin Medical University, Tianjin 300070, P.R. China
}

Received July 6, 2014; Accepted July 21, 2015

DOI: $10.3892 / 01.2015 .3899$

\begin{abstract}
Primary neuroendocrine carcinoma (NEC) of the ureter is a rare entity, with few cases reported in the literature. These tumors are usually locally aggressive and are associated with a poor prognosis due to the thin walls of the ureter. This is the case report of a 69-year-old male patient who presented with left flank pain and gross hematuria. Following diagnosis of ureteral carcinoma, nephroureterectomy was performed. Postoperatively, the patient refused chemotherapy and radiotherapy, and succumbed to extensive metastases after 12 months of follow-up. The aim of the present study was to provide a literature review of NEC and its clinical and pathological characteristics, diagnostic methods and latest modifications in therapy.
\end{abstract}

\section{Introduction}

Neuroendocrine carcinomas (NECs) comprise a heterogeneous group of neoplasms, which arise from peptide- or amine-producing endocrine cells throughout the body (1). This type of tumor is mainly encountered in the lungs. Extrapulmonary counterparts are rarely encountered, although they have been described in a variety of organs, including the esophagus, stomach, pancreas, gallbladder, uterine cervix, kidney, urinary bladder and prostate gland (2). To the best of our knowledge, there are only few case reports of NECs located in the genitourinary tract, with the majority of cases reported in the bladder, whereas tumors originating from the ureter are extremely rare (3). The histogenesis of this type of tumor has not been fully elucidated, although several hypotheses have been suggested. Furthermore, due to its rarity, the clinical behavior and theory underlying the development of primary NEC of the ureter have not been well established. The

Correspondence to: Professor Hongshun Ma, Department of Urology, Tianjin First Central Hospital, Building A, 24 Fukang Road, Nankai District, Tianjin 300912, P.R. China

E-mail: mahongshun@hotmail.com

Key words: neuroendocrine carcinoma, ureter, clinical characteristics, pathological characteristics, management aim of the present study was to report the clinical and pathological characteristics and management of a case of NEC with combined small-cell and atypical carcinoid components. In the light of the present case report, a recent systematic literature review of the clinical presentation and management of this rare tumor was also performed. Written informed consent was obtained from the patient's family.

\section{Case report}

A 69-year-old Chinese Han male patient was admitted to the Department of Urology of Tianjin First Central Hospital (Tianjin, China) with a 10-month history of gross hematuria and 1-month history of left flank pain. The medical history of the patient included 17 years of hypertension, 20 years of coronary heart disease and an allergy to iodine. The findings of the physical examination were normal. The findings of the routine laboratory examinations were unremarkable. However, on urinalysis, there was macroscopic hematuria and 40-50 white blood cells per high-power field. On magnetic resonance imaging of the urinary tract, the iliac wing level around the left ureter exhibited an iso-intense T2 signal, an irregular mass with a rough outline and unclear boundaries of the corresponding level of the left iliac vessels. There was occlusion of the left ureter at the corresponding level, with hydronephroureterosis above the mass and undevelopment of the segment of the left ureter below the mass (Figs. 1,2 and 3). The right kidney was normal. Chest radiographs were performed and revealed no primary or metastatic lung lesions. Left nephroureterectomy was performed and the clinical diagnosis was primary ureteral tumor. The gross examination revealed an ovoid, solid, white mass, sized $3.5 \times 3.0 \times 1.6 \mathrm{~cm}$, originating from the ureteral mucosa and protruding into the ureteral lumen, with invasion of the periureteral adipose tissue. Examination under a light microscope revealed that the tumor was composed of atypical carcinoid cells with diverse shapes, with common mitotic figures, and small cells with a round to fusiform shape, scant cytoplasm, fine granular nuclear chromatin, and absent or inconspicuous nucleoli (Fig. 4). The tumor cells exhibited cytoplasmic positivity for cytokeratin (CK)7, epithelial membrane antigen (EMA), CD56 and synaptophysin, and negativity for neuron-specific enolase (NSE), chromogranin A (CgA) and CK20. The Ki-67/MIB1 index was 20\%. The patient was diagnosed with NEC of the ureter with atypical carcinoid and small-cell carcinoma components. The postoperative recovery 


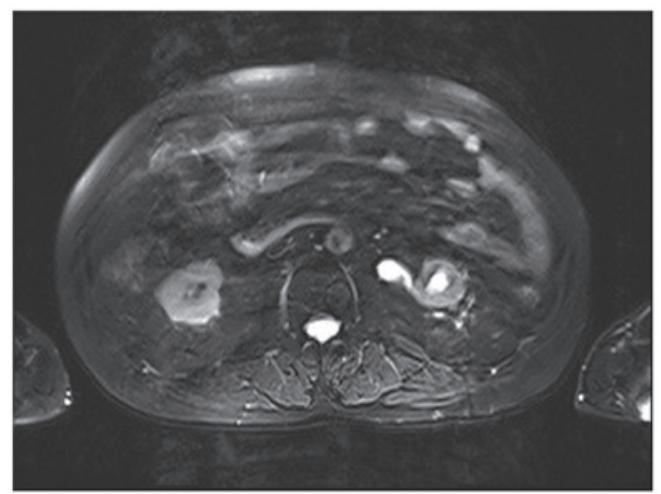

Figure 1. Occlusion of the left ureter, with hydronephroureterosis above the mass.

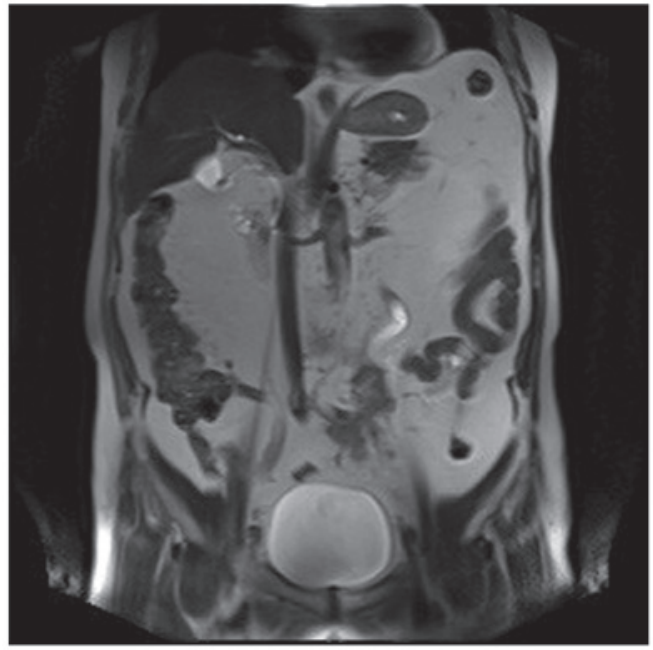

Figure 2. The iliac wing level around the left ureter exhibiting an iso-intense T2 signal and an irregular mass with a rough outline.

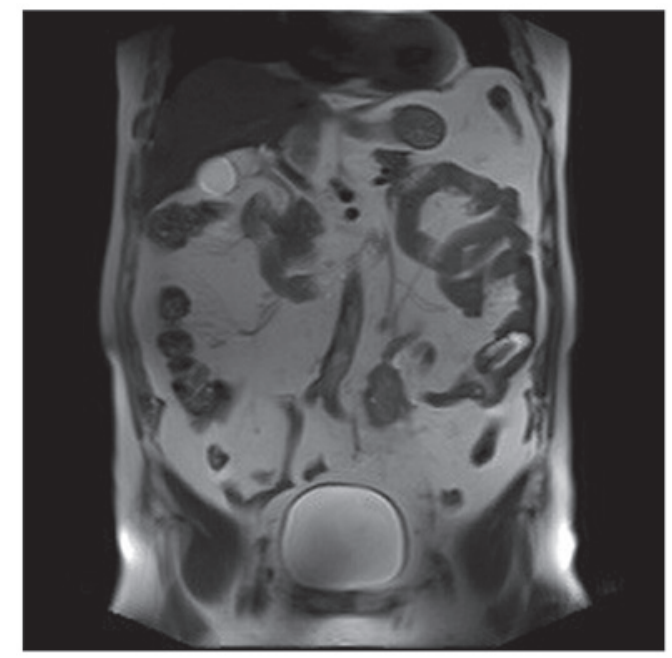

Figure 3. The segment of the left ureter below the mass was undeveloped.

of the patient was uncomplicated; however the patient refused chemotherapy or radiotherapy. During the regular follow-up examinations, there was no evidence of tumor recurrence at 5 months postoperatively; however, he succumbed to extensive metastases after 12 months of follow-up.

\section{Discussion}

The types of NEC include large-cell NEC, giant-cell NEC, small-cell carcinoma, carcinoid and atypical carcinoid tumors. The term 'NEC' describes a heterogeneous group of tumors 

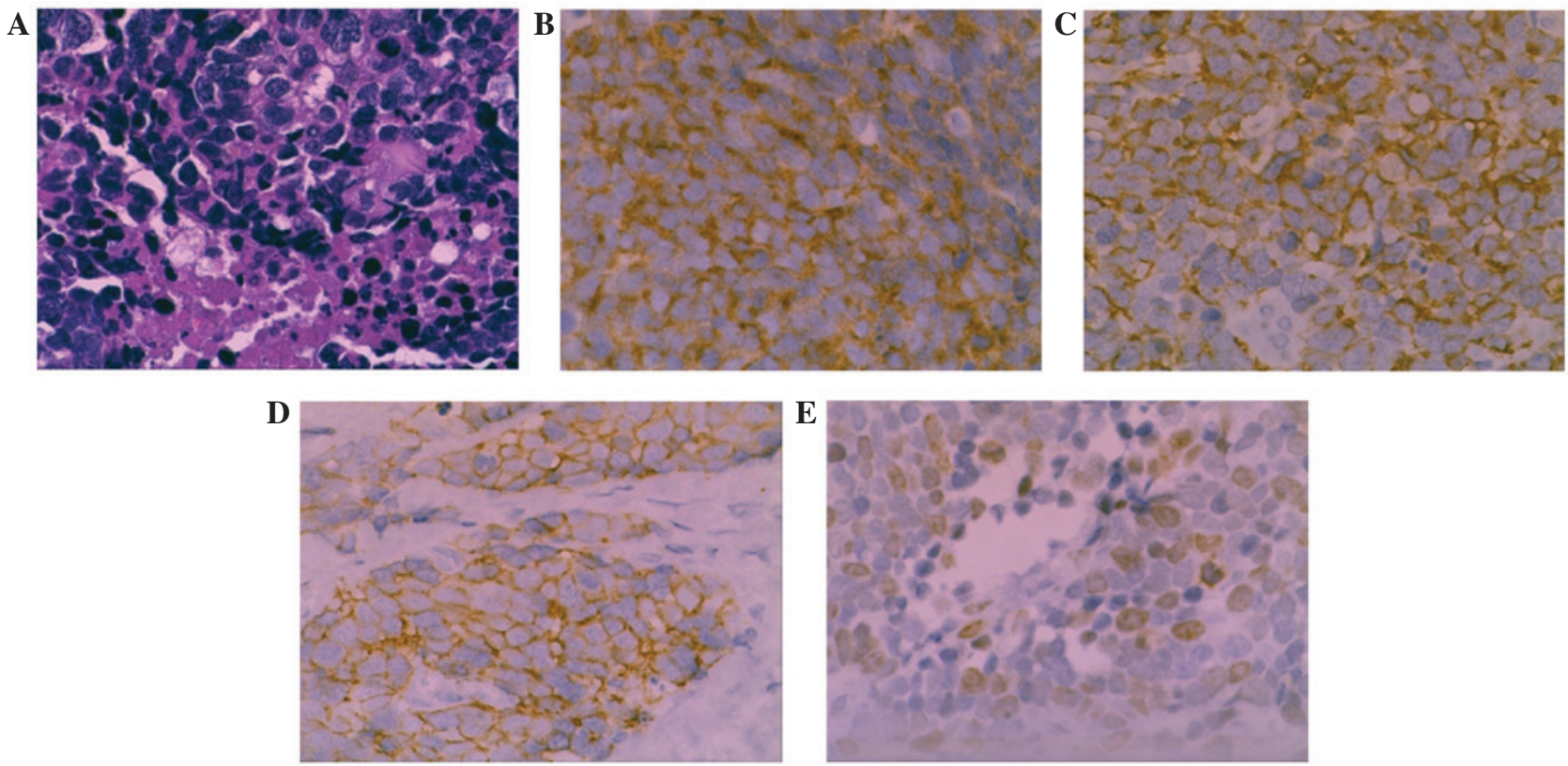

Figure 4. (A) The tumor is composed of atypical cells with diverse shapes, with common mitotic figures, and small cells with a round to fusiform shape and hyperchromatic nuclei (hematoxylin and eosin staining; magnification, x400). Positive immunohistochemical staining for (B) synaptophysin, (C) cytokeratin 7 and (D) CD56 (magnification, x400). (E) The Ki-67/MIB1 index was 20\% (magnification, x400).

originating from neuroendocrine cells in different organs. Approximately $74 \%$ of these tumors originate in the gastrointestinal tract and $10 \%$ originate in the lungs, whereas the remainder are scattered in various systems (1). NECs arising from the urinary tract are extremely rare and represent $<0.5 \%$ of urinary tract tumors (3). Among these, primary NECs of the kidney, ureter, bladder and prostate are the most commonly reported, with the majority originating in the bladder, while primary NECs originating in the ureter are extremely rare. Due to the low incidence of ureteral NECs, these tumors are poorly understood and their origin remains controversial. The following four hypotheses have been suggested for the origin of the tumors: i) The urothelium with neuroendocrine differentiation; ii) neuroendocrine cells present in the urinary tract; iii) the entrapped neural crest in the ureter during embryogenesis; and iv) undifferentiated stem cells that differentiate towards a urothelial or squamous cell lineage $(4,5)$.

NECs of the ureter are similar to other urothelial carcinomas. The most common symptoms include intermittent painless gross or microscopic hematuria, blunt pain in the lower back in a proportion of the patients, whereas a few patients present with the syndrome of inappropriate antidiuretic hormone secretion. The diagnosis mainly relies on histology. NECs do not usually consist of more than a single tissue type (6). Similar to other types of NEC, the pathological characteristics of urinary NEC are as follows: i) Large-cell NEC consists of large cells typically exhibiting significant pleiomorphism, large nuclei with coarse and granular chromatin and prominent nucleoli, often with significant mitotic activity and palisading with rosette formation with a sheet-like and trabecular growth patterns, usually with extensive necrosis (7); ii) giant-cell NEC mainly consists of larger cancer cells of different forms, multinucleated giant cells, common mitotic figures and extensive necrosis (8); iii) small-cell carcinoma consists of small cells of uniform shape, with little or no cytoplasm, round nuclei, unclear cell boundaries, forming flaky or nested cell masses, with large areas of necrosis; iv) carcinoids consist of cells that are histologically medium-sized with consistent shape and size, round or oval centrally located nuclei, with rare or absent mitotic figures; the cells are arranged in nests, islets, trabeculae, or cords, with palisading; and v) atypical carcinoids exhibit microscopic characteristics similar to carcinoids, although with greater nuclear atypia, higher mitotic activity and focal necrosis. The overall microscopic morphology is inconsistent, with atypical characteristics and common mitotic figures (2-4/10 high-power field), with the cancer cells arranged in trabeculae, cords or nests. Suspected cases should undergo immunohistochemical examination, including the detection of CKs and EMA, which contribute to the diagnosis of the disease.

Ureteral small-cell carcinoma, large-cell NEC and giant-cell NEC are the most highly malignant among these five types of NECs, exhibiting strong invasiveness and poor prognosis. We herein report a case of small-cell carcinoma combined with of atypical carcinoid characteristics. The patient refused chemotherapy and radiotherapy following surgery, and succumbed to the disease after 12 months of follow-up. The treatment principle for this disease is the use of combined therapies with surgical excision. If the tumor is completely surgically resectable, it may significant improve the survival rate and quality of life. The surgical method usually selected is open radical resection with excision of a bladder cuff. As the tumor is often larger and may have already metastasized by the time the patient seeks a consultation, radical resection should be performed as early as possible. In addition, due to the advanced stage and early micro-metastases, tumors often cannot be completely removed; therefore, radical surgical resection alone usually does not prevent disease progression. Chemotherapy and radiotherapy following surgery are often required for effective treatment. However, the treatment modalities (surgery, chemotherapy and 
radiotherapy) are not optimally defined due to the rarity of these tumors. A potential benefit has been suggested with adjuvant chemotherapy. Treatment of disseminated disease is based on chemotherapy with a platinum agent (9-11).

NECs are aggressive tumors that usually present at an advanced stage. The diagnosis mainly relies on pathological characteristics. The prognosis is poorer compared with that of urothelial cancer. Due to the lack of prospective studies, there is currently no standard therapeutic approach. For localized disease, aggressive surgical treatment combined with adjuvant chemotherapy may prolong overall and disease-free survival. For metastatic disease, chemotherapy using a platinum agent is currently the mainstay of treatment.

\section{References}

1. Volante M, Rindi G and Papotti M: The grey zone between pure (neuro) endocrine and non-(neuro) endocrine tumours: a comment on concepts and classification of mixed exocrine-endocrine neoplasms. Virchows Arch 449: 499-506, 2006.

2. Kim JH, Lee SH, Park J, et al: Extrapulmonary small-cell carcinoma: a single-institution experience. Jpn J Clin Oncol 34: 250-254, 2004.

3. Ping JH, Chen ZX, Jiong Q, Han YQ and Nong X: Small cell neuroendocrine carcinoma of the ureter: A case report and literature review. Oncol Lett 7: 728-730, 2014.
4. Banerji JS, Korula A and Panicker JB: Multicentric small cell neuroendocrine neoplasm of the renal pelvis and ureter with concomitant focal high-grade urothelial carcinoma of the ureter: A case report. Indian J Urol 24: 571-574, 2008.

5. Fetissof F, Dubois MP, Lanson Y and Jobard P: Endocrine cells in renal pelvis and ureter, an immunohistochemical analysis. J Urol 135: 420-421, 1986.

6. Kim TS, Seong DH and Ro JY: Small cell carcinoma of the ureter with squamous cell and transitional cell carcinomatous components associated with ureteral stone. J Korean Med Sci 16: 796-800, 2001.

7. Shin MK, Choi CM, Oh YJ and Kim, NI: CK20 Positive Large-cell neuroendocrine carcinoma presenting with skin metastases. Ann Dermatol 23 (Suppl 1): S20-S24, 2011.

8. Dundr P, Pesl M, Povýsil C, Vítková I and Dvorácek J: Large cell neuroendocrine carcinoma of the urinary bladder with lymphoepithelioma-like features. Pathol Res Pract 199: 559-563, 2003.

9. Debry C, Rouyer N, Grandjean E, Gentine A, Stierle JL and Conraux C: Neuroendocrine giant-cell epithelioma. Description of a clinical case and review of the literature. Ann Otolaryngol Chir Cervicofac 109: 345-350, 1992 (In French).

10. Coelho HM, Pereira BA and Caetano PA: Large cell neuroendocrine carcinoma of the urinary bladder: case report and review. Curr Urol 7: 155-159, 2013.

11. Ahsaini M, Riyach O, Tazi MF, et al: Small cell neuroendocrine carcinoma of the urinary tract successfully managed with neoadjuvant chemotherapy. Case Rep Urol 2013: 598325, 2013. 